\title{
Influência do tamanho de parcelas experimentais na seleção de progênies de E. camaldulensis Dehnh.
}

\author{
Influence of the size plots in the selection of progenies \\ of the Eucalyptus camaldulensis Dehnh.
}

\author{
William de Medeiros Silva', Mario Luiz Teixeira de Moraes², Silvelise Pupin ${ }^{3}$, \\ Janete Motta da Silva ${ }^{4}$, Alexandre Magno Sebbenn ${ }^{5}$ e Bruno Ettore Pavan ${ }^{6}$
}

\section{Resumo}

$\mathrm{Na}$ escolha do tamanho da parcela experimental, inúmeros fatores devem ser considerados no sentido de aumentar a eficiência da seleção com o menor custo operacional possível. Este trabalho teve como objetivo verificar a influência de dois tamanhos de parcelas experimentais em testes de progênies de $E$. camaldulensis, para as variáveis forma de fuste, DAP, altura total das árvores e volume total das árvores. EXP 1 e EXP 2 foram implantados em delineamento de blocos casualizados, com 133 progênies e espaçamento de 3,0 $\mathrm{m} \times 1,8 \mathrm{~m}$. O EXP 1 foi composto por parcelas experimentais de 5 plantas e quatro repetições e no EXP 2 utilizou-se uma planta por parcela e 20 repetições. As estimativas de componentes de variância e parâmetros genéticos foram obtidas utilizando o método REML/BLUP. O coeficiente de variação genotípica entre as progênies foi semelhante nos dois experimentos para forma de fuste e superior para as variáveis DAP, altura total das árvores e volume total das árvores no EXP 2. Para os quatro caracteres, a herdabilidade em nível de média de progênies foi maior no EXP 2, indicando maior eficiência na seleção. No EXP 2 ocorreram maiores ganhos com a seleção entre progênies, indicando um favorecimento deste delineamento aos indivíduos superiores de uma forma geral. A adoção de experimentos com uma planta por parcela aliada ao grande número de repetições é recomendado para testes de progênies da espécie Eucalyptus camaldulensis.

Palavras-chave: Parâmetros genéticos, delineamento experimental, REML/BLUP.

\begin{abstract}
In the choice of the size plots numerous factors must be considered in order to increase the efficiency of its selection, with the lowest operating cost. This work aimed to study the influence of two sizes plots in E. camaldulensis progeny tests for the variables stem shape, diameter at breast height, total height of the tree and total volume of the tree. EXP 1 and EXP 2 were implanted in a randomized block design, with 133 progenies and spacing of $3.0 \mathrm{mx} 1.8 \mathrm{~m}$. The EXP 1 set up five plants per plot and four replications and EXP 2 used a plant per plots and 20 repetitions. Estimates of variance components and genetic parameters were obtained using the REML / BLUP method. Coefficient of genotypic variation among progenies was similar in both experiments to stem shape and superior to the diameter at breast height, total height in EXP 2. For the four traits, heritability average level of progeny was higher in EXP 2, indicating greater efficiency in the genetic control at average progenies level. Experiment with one plant per plot offered higher gain
\end{abstract}

\footnotetext{
${ }^{1}$ Doutorando no Departamento de Produção Vegetal. UNESP - Universidade Estadual Paulista Júlio de Mesquita Filho / Faculdade de Ciências Agrárias e Veterinárias de Jaboticabal. Via de Acesso Prof. Paulo Donato Castellane, s/n - Rural 14884900 - Jaboticabal, SP, Brasil.

2Professor Titular do Departamento de Fitotecnia, Tecnologia de Alimentos e Sócio Economia. UNESP - Universidade Estadual Paulista Júlio de Mesquita Filho / Faculdade de Engenharia de Ilha Solteira. Avenida Brasil, 56 - Centro - 15385000 - Ilha Solteira, SP, Brasil.

${ }^{3}$ Doutoranda no Departamento de Fitotecnia, Tecnologia de Alimentos e Sócio Economia. UNESP - Universidade Estadual Paulista Júlio de Mesquita Filho / Faculdade de Engenharia de Ilha Solteira. Avenida Brasil, 56 - Centro - 15385-000 - Ilha Solteira, SP, Brasil. E-mail: silvelise.pupin@gmail.com

${ }^{4}$ Doutora pelo Departamento de Fitotecnia, Tecnologia de Alimentos e Sócio Economia. UNESP - Universidade Estadual Paulista Júlio de Mesquita Filho / Faculdade de Engenharia de Ilha Solteira. Avenida Brasil, 56 - Centro - 15385-000 - Ilha Solteira, SP, Brasil. E-mail: janetemottasilva@gmail.com.

${ }^{5}$ Pesquisador Doutor. IF - Instituto Florestal de São Paulo / Estação Experimental de Tupi. Rodovia Luiz de Queiroz, Km 149,5 - Caixa-postal: 339 - Tupi - 13400-970 - Piracicaba, SP, Brasil. E-mail: alexandresebbenn@yahoo.com.br.

${ }^{6}$ Professor Adjunto do Departamento de Fitotecnia, Tecnologia de Alimentos e Sócio Economia. UNESP - Universidade Estadual Paulista Júlio de Mesquita Filho / Faculdade de Engenharia de Ilha Solteira. Avenida Brasil, 56 - Centro - 15385000 - Ilha Solteira, SP, Brasil. E-mail: pavan@agr.feis.unesp.br.
} 
Silva et al. - Influência do tamanho de parcelas experimentais

na seleção de progênies de E. camaldulensis Dehnh

with progenies selection, indicating favor on this design for superior individuals in general. The adoption of experiments with one plant per parcel together with the large number of replication is recommended for the Eucalyptus camaldulensis progeny tests.

Keywords: progeny test, experimental design, REML / BLUP.

\section{INTRODUÇÃO}

Os plantios de eucalipto totalizam mais de 20 milhões de hectares no mundo (BOOTH, 2013) e são importantes devido à crescente demanda por produtos madeireiros e por representar a principal fonte de matéria-prima para as indústrias à base de celulose de fibra curta. No Brasil, as áreas plantadas com eucalipto totalizaram 5,56 milhões de hectares em 2014, equivalente a 71,9\% das florestas plantadas (IBA, 2015).

O Eucalyptus camaldulensis Dehnh. foi uma das primeiras espécies do gênero a ser plantada com sucesso em países além da Austrália (ELDRIDGE et al., 1993). No Brasil, apesar de ter sido introduzida no início do século passado, esta espécie assumiu maior relevância quando os maciços florestais expandiram-se das regiões Sul e Sudeste para o Centro-Oeste e Nordeste, adaptando-se melhor às condições de alta temperatura e longo período seco, que eram inadequadas para as espécies mais utilizadas, como E. grandis, E. saligna e E. urophylla (GOLFARI et al., 1978).

Eucalyptus camaldulensis é caracterizado por sua rusticidade e reconhecido por ser uma espécie alvo em programas de silvicultura clonal interespecífica, garantindo aos seus híbridos boa adaptabilidade a diversas condições e regiões edafoclimáticas (SILVA, 2010). Sua ampla variabilidade genética entre e dentro de procedências, possibilitou consideráveis avanços genéticos para a produção, justificando sua adoção em programas de melhoramento genético. No entanto, existem poucos relatos de sua utilização em plantios comerciais, ficando restrito a poucas empresas e a áreas experimentais (QUISEN, 2007). Dessa forma, o potencial desta espécie ainda é pouco aproveitado, mas pode ser substancial frente aos desafios vindouros, como mudanças climáticas e expansão de áreas agrícolas, com grandes restrições ao plantio.

Porém, para um melhor aproveitamento deste potencial, programas de melhoramento bem delineados são fundamentais, e podem possibilitar a exploração das potencialidades do E. camaldulensis. Dentre os diversos instrumentos para auxiliar o trabalho do melhorista, os testes de progênies são indispensáveis, sendo utilizados para estimar parâmetros genéticos e conduzir a seleção de famílias e/ou indivíduos, quantificando a magnitude e natureza da variância genética, a fim de maximizar os ganhos genéticos (ARANTES et al., 2010).

Entretanto, a simples adoção dos testes de progênies não garante o sucesso de um programa de melhoramento. Medidas como a utilização de preditores adequados (BAUER et al., 2006; FARIAS NETO et al., 2009; VERARDI et al., 2009; PUPIN et al., 2015), procedimentos para seleção precoce (MASSARO et al, 2010; PINTO et al. 2014; PAVAN et al., 2014) e tamanho e formatos adequados de parcelas (SILVA et al., 2003; OLIVEIRA et al. 2011; DIAS, 2014), podem aumentar a eficiência da seleção. Dentre as ferramentas citadas, a utilização de tamanho e forma de parcelas vem sendo pouco explorada em teste de progênies, estando seus estudos mais concentrados em testes clonais (SILVA et al., 2003; SCARPINATI et al., 2009; ARAÚJO et al., 2015).

Em experimentos florestais, fatores como altos custos envolvidos na experimentação, exigência e disponibilidade de grandes áreas experimentais, amplo número de tratamentos e a heterogeneidade do sítio de instalação, reforçam a importância de estudos que abordem o tamanho das parcelas (DIAS, 2014). Nesse tipo de experimento, costuma-se usar parcelas com números reduzidos de plantas, o que permite a adoção de blocos com menor heterogeneidade, além de possibilitar um maior número de repetições, com o mesmo material disponível, gerando estimativas de maior confiabilidade (RESENDE, 1995), uma vez que maior número de repetições tende a gerar maiores valores de acurácia (RESENDE; DUARTE, 2007). No entanto, testes com poucas plantas por parcela geram um alto nível de competição intergenotípica (PAVAN et al., 2012), que não deve ser ignorada. Dessa forma, a escolha do tamanho da parcela experimental não é uma decisão simples, devendo-se levar em conta inúmeras variáveis envolvidas, possibilitando uma seleção eficiente, com alto grau de acerto e menor custo operacional possível. 
Nesse contexto, o objetivo deste trabalho foi avaliar dois testes de progênies de E. camaldulensis com diferentes tamanhos de parcelas experimentais, e a influência destes na forma do fuste e em três caracteres de crescimento (DAP, altura total da árvore e volume), assim como nas respectivas estimativas de parâmetros genéticos.

\section{MATERIAL E MÉTODOS}

Foram avaliadas famílias de polinização aberta de E. camaldulensis, em dois testes de progênies com diferentes tamanhos de parcelas experimentais. Os experimentos foram instalados em outubro de 2008, na Fazenda de Ensino, Pesquisa e Extensão, da Faculdade de Engenharia de Ilha Solteira (FEIS/UNESP), localizada no município de Selvíria - MS, nas coordenadas geográficas $20^{\circ} 20^{\prime} 25^{\prime \prime}$ de latitude Sul e $51^{\circ} 24^{\prime} 33^{\prime \prime}$ de longitude Oeste, a $371 \mathrm{~m}$ acima do nível do mar. Nos dois experimentos utilizou-se o delineamento de blocos casualizados, com 133 tratamentos (progênies) e espaçamento de 3,0 $\mathrm{m} \times 1,8 \mathrm{~m}$. O primeiro experimento foi composto por parcelas experimentais de 5 plantas e quatro repetições (EXP1), no segundo experimento utilizou-se uma planta por parcela e 20 repetições (EXP2). As árvores que constituem os testes de progênies são provenientes da polinização livre entre 250 árvores selecionadas em uma população base de Eucalyptus camaldulensis, originária de 25 matrizes procedentes da Austrália (SILVA, 2010).

Aos seis anos de idade (2014) foram mensurados os caracteres de crescimento: diâmetro a altura do peito (DAP, $\mathrm{cm})$ e altura total de árvores (ALT, m). A partir dessas variáveis, obteve-se o volume total com casca das árvores $\left(\mathrm{VOL}, \mathrm{m}^{3}\right)$ utilizando a seguinte fórmula:

$$
V=\pi *\left(\frac{D A P^{2}}{40000}\right) *(A L T * 0,5)
$$

Concomitantemente, foi avaliada a forma de fuste das árvores, com base em uma escala de notas de 1 (muito bifurcada) a 7 (sem bifurcações) para bifurcação das árvores, e outra escala para tortuosidade das árvores com notas de 1 (tortuosidade acentuada) a 7 (sem tortuosidade). As notas dadas para bifurcação e tortuosidade das árvores foram somadas gerando notas de 2 a 14 para a variável forma de fuste.

As estimativas dos componentes de variância e predição dos parâmetros genéticos foram obtidos para cada variável. Foram empregadas análises individuais e conjunta para os dois testes de progênies, utilizando-se o procedimento REML/BLUP (máxima verossimilhança restrita/melhor predição linear não viciada), disponível no software genético-estatístico SELEGEN, desenvolvido por Resende (2007). Para a análise individual do EXP 1 assumiu-se as progênies como sendo de meios-irmãos com várias observações por parcela, seguindo o modelo proposto por Resende (2007): $y=X r+Z a$ $+W p+e$, em que $y$ é o vetor de dados, $r$ é o vetor dos efeitos de repetição (assumidos como fixos) somados à média geral, $a$ é o vetor dos efeitos genéticos aditivos individuais (aleatórios), $p$ é o vetor dos efeitos de parcelas (aleatórios), e é o vetor de erros ou resíduos (aleatórios). As letras maiúsculas representam as matrizes de incidência para os referidos efeitos. Na análise individual do EXP 2 assumiu-se as progênies como sendo de meios-irmãos com uma observação por parcela, seguindo o modelo proposto por Resende (2007): $y=X r+Z a+e$, que é semelhante ao primeiro modelo porém sem o vetor $p$. A análise conjunta dos experimentos foi realizada com base no modelo estatístico (RESENDE, 2007): $y=X r+Z a+W p+T i+e$, em que $y$ é o vetor de dados, $r$ é o vetor dos efeitos de repetição (assumidos como fixos) somados à média geral, $a$ é o vetor dos efeitos genéticos aditivos individuais (assumidos como aleatórios), p é o vetor dos efeitos de parcela (assumidos como aleatórios), $i$ é vetor dos efeitos da interação genótipo $\mathrm{x}$ ambiente (aleatórios) e $e$ é o vetor de erros ou resíduos (aleatórios). As letras maiúsculas representam as matrizes de incidência para os referidos efeitos. Segundo Resende (2007), esse modelo pode ser usado para analisar delineamentos com diferentes tamanhos de parcela pois ajusta os efeitos de parcela, entretanto, para os dados advindos do experimento com uma só planta por parcela, apenas um código de parcela foi usado para todos os dados. Este procedimento é estatisticamente correto pois ajusta nenhum efeito de parcela para os experimentos com uma planta por parcela uma vez que toda informação associada ao código dado a parcela, será, nesse caso, sugada pelo efeito fixo associado ao tipo de delineamento quanto ao tamanho de parcela (RESENDE, 2007).

Os parâmetros genéticos, componentes de variância e estatísticas descritivas estudados foram: $\sigma_{\mathrm{a}}^{2}$ : variância genética aditiva; $\sigma_{\text {parc }}^{2}$ : variância ambiental entre parcelas; $\sigma_{\mathrm{e}}^{2}$ : variância residual (am- 
biental + não aditiva); $h^{2}$ : herdabilidade individual no sentido restrito, ou seja, dos efeitos aditivos; $c^{2}{ }_{\text {int }}$ : coeficiente de determinação dos efeitos da interação genótipos x ambientes; $h^{2}{ }_{\mathrm{mp}}$ : herdabilidade da média de progênies, assumindo sobrevivência completa; $a c_{\text {prog: }}$ : acurácia da seleção de progênies, assumindo sobrevivência completa; $r_{\text {gloc }}$ : correlação genotípica entre o desempenho das progênies nos dois ambientes; $h^{2}$ ad herdabilidade aditiva dentro de parcela; $C V_{\mathrm{gp}} \%$ : coeficiente de variação genotípica entre progênies; MG: Média geral do experimento; Amplitude dos valores genotípicos de indivíduos; GS/GS(\%): ganho com a seleção; $N_{e}$ : tamanho efetivo; $\rho$ : coeficiente de correlação de Spearman e FC: número de famílias coincidentes entre as 30 melhores. Para o ganho de seleção adotou-se a seleção individual dos melhores indivíduos pelo valor genético aditivo com uma intensidade de seleção de 33\%, visando selecionar os melhores indivíduos e manter uma ampla base genética.

\section{RESULTADOS E DISCUSSÃO}

O erro experimental para as quatro variáveis estudadas foi menor no experimento de uma planta por parcela, indicando que em termos de eficiência experimental, o EXP2 foi mais eficiente em relação ao EXP1 (Tabela 1). Este resultado já era esperado, uma vez que ao aumentar o número de repetições, em função da diminuição do número de plantas por parcela, a distribuição dos indivíduos de cada família na área experimental se torna mais homogênea. Pavan et al. (2012), ao avaliarem o efeito competicional em testes de progênies de eucalipto, encontraram resultados contrastantes com os deste trabalho para o erro experimental, atrelando os resultados ao efeito da competição.

Comparando as análises experimentais, verificou-se que a variância genética aditiva $\left({ }_{\mathrm{a}}{ }^{2}\right)$ foi maior no EXP1 em relação ao EXP2 para a variável forma de fuste. Entretanto, o EXP2 apresentou maiores valores para as outras três variáveis (DAP, ALT e VOL) em relação ao EXP1 (Tabela 1). De acordo com Scarpinati et al. (2009), isso se justifica pelo fato de que à medida que a competição entre as famílias é intensificada por meio da disposição das parcelas, aumentam-se as diferenças entre elas, e como consequência, aumenta-se também a variância genética.

Tabela 1. Componentes de variância e estimativas de parâmetros genéticos para forma do fuste, diâmetro a altura do peito (DAP), altura total da árvore (ALT) e volume total da árvore (VOL) para os testes de progênies de Eucalyptus camaldulensis com cinco plantas por parcelas (EXP 1) e uma planta por parcela (EXP 2).

Table 1. Variance components and estimates of genetic parameters for stem form, breast height diameter (DAP), total height of the tree (ALT) and total volume of the tree (VOL) for progeny tests of Eucalyptus camaldulensis with five plants per plots (EXP 1) and a plant per plot (EXP 2).

\begin{tabular}{|c|c|c|c|c|c|c|c|c|}
\hline \multirow{2}{*}{$\begin{array}{l}\text { Componentes } \\
\text { de Variância }\end{array}$} & \multicolumn{2}{|c|}{ FORMA FUSTE } & \multicolumn{2}{|c|}{ DAP } & \multicolumn{2}{|c|}{$\overline{A L T}$} & \multicolumn{2}{|c|}{ VOL } \\
\hline & EXP 1 & EXP 2 & EXP 1 & EXP 2 & EXP 1 & EXP 2 & EXP 1 & EXP 2 \\
\hline$\overline{\sigma_{a}^{2}}$ & 0.843 & 0.742 & 1.785 & 4.131 & 3.392 & 5.982 & 0 & 0.002 \\
\hline$\sigma_{\text {parc }}^{2}$ & 0.526 & - & 0.051 & - & 0.612 & - & 0 & - \\
\hline$\sigma_{e}^{2}$ & 4.386 & 4.026 & 8.923 & 8.001 & 13.618 & 12.352 & 0.005 & 0.005 \\
\hline$h^{2}{ }_{\mathrm{a}}^{\mathrm{e}}$ & $0.14 \pm 0.04$ & $0.16 \pm 0.04$ & $0.16 \pm 0.04$ & $0.34 \pm 0.06$ & $0.19 \pm 0.04$ & $0.32 \pm 0.06$ & $0.16 \pm 0.04$ & $0.37 \pm 0.06$ \\
\hline$h_{m p}^{2}$ & 0.35 & 0.45 & 0.45 & 0.65 & 0.46 & 0.64 & 0.45 & 0.67 \\
\hline$h^{2}{ }_{\text {ad }}$ & 0.12 & 0.12 & 0.13 & 0.27 & 0.15 & 0.26 & 0.12 & 0.31 \\
\hline$A c_{\text {prog }}$ & 0.59 & 0.67 & 0.67 & 0.8 & 0.67 & 0.8 & 0.67 & 0.81 \\
\hline$C \mathrm{v}_{\mathrm{gp}} \%$ & 5.32 & 4.76 & 5.51 & 8.26 & 5.38 & 6.72 & 13 & 21.33 \\
\hline$c^{2}{ }_{\text {int }}$ & \multicolumn{2}{|c|}{0.0019} & \multicolumn{2}{|c|}{0.0101} & \multicolumn{2}{|c|}{0.0041} & \multicolumn{2}{|c|}{0.0148} \\
\hline$r_{\text {gloc }}$ & \multicolumn{2}{|c|}{0.95} & \multicolumn{2}{|c|}{0.84} & \multicolumn{2}{|c|}{0.93} & \multicolumn{2}{|c|}{0.79} \\
\hline MG & 8.62 & 9 & 12.12 & 12.3 & 17.1 & 18.19 & 0.11 & 0.12 \\
\hline Amplitude & 2.56 & 3 & 4.5 & 12.18 & 5.97 & 9.52 & 0.09 & 0.39 \\
\hline GS & 0.43 & 0.45 & 0.7 & 1.4 & 0.99 & 1.6 & 0.01 & 0.03 \\
\hline GS(\%) & 4.98 & 4.96 & 5.77 & 11.38 & 5.78 & 8.79 & 9.09 & 25 \\
\hline $\mathrm{NE}$ & 246.46 & 238.36 & 229.62 & 247.58 & 227.44 & 235.73 & 226.97 & 250.6 \\
\hline$\rho$ & \multicolumn{2}{|c|}{0.43} & \multicolumn{2}{|c|}{0.47} & \multicolumn{2}{|c|}{0.52} & \multicolumn{2}{|c|}{0.5} \\
\hline $\mathrm{FC}$ & \multicolumn{2}{|c|}{13} & \multicolumn{2}{|c|}{17} & \multicolumn{2}{|c|}{15} & \multicolumn{2}{|c|}{16} \\
\hline
\end{tabular}

$\sigma^{2}$ : variância genética aditiva. $\sigma^{2}$ : variância ambiental entre parcelas, $\sigma^{2}:$ variância residual (ambiental + não aditiva), $h^{2}:$ herdabilidade individual no sentido restrito, $h^{2}$ mp : herdabilidade da média de progênies, $h^{2}$ ad: herdabilidade aditiva dentro de parcela, $A c_{\text {prog }}$ : acurácia da seleção de progênies, $C V$ gp\%: coeficiente de variação genotípica entre progênies, $c^{2}$ int: coeficiente de determinação dos efeitos da interação genótipos $\mathrm{X}$ ambientes, $r_{\text {gloc }}$ : correlação genotípica entre o desempenho das progênies nos vários ambientes,MG: Média geral do experimento, Amplitude: amplitude do valores genotípicos entre os indivíduos, GS: ganho com a seleção, $\mathrm{N}_{\mathrm{e}}$ : tamanho efetivo, $\rho$ : coeficiente de correlação de Spearman e FC: número de famílias coincidentes entre as 30 melhores. 
Os coeficientes de variação genotípica entre progênies $\left(\mathrm{CV}_{\mathrm{gp}} \%\right)$ variaram de $4,76 \%$ (forma de fuste - EXP2) a 21,33\% (volume - EXP2), indicando presença de variância genética entre as progênies (Tabela 1). Coeficientes de variação genética acima de 7\% são considerados altos por Sebbenn et al. (1998). A exemplo da $\sigma^{2}{ }_{a}, \mathrm{o} \mathrm{CV}_{\mathrm{gp}} \%$ foi semelhante nos dois experimentos para a variável forma de fuste, apresentando uma diferença de 0,56\% entre as estimativas dos dois experimentos, enquanto que para os outros caracteres, os valores encontrados no EXP2 foram consideravelmente superiores. Em função disso pode-se afirmar que a diminuição do tamanho da parcela não interferiu na conformação do fuste das progênies, porém apresentou um efeito considerável sobre os caracteres de crescimento, intensificando as variâncias e amplitudes de tais caracteres. O mesmo efeito sobre as variâncias e amplitudes foi encontrado por Scarpinati et al. (2009) e Araújo et al. (2015), ambos avaliando diferentes arranjos experimentais em clones de eucalipto.

A herdabilidade em nível de médias de progênies $\left(h^{2}{ }_{m p}\right)$, para os quatro caracteres estudados, tanto no EXP1 como no EXP2, foi maior do que a herdabilidade individual no sentido restrito $\left(h^{2}\right)$ (Tabela 1). De acordo com Vencovsky e Barriga (1992), isso tende a acontecer quando se minimizam os efeitos ambientais pelo número de repetições e de plantas por parcela. Portanto, a seleção pode ser mais eficiente com base nas médias de progênies do que em plantas individuais. Ao comparar os dois experimentos, nota-se que a $h_{m p}{ }_{m p}$ para os quatro caracteres estudados, foi maior no EXP2 do que no EXP1, sugerindo que um número maior de repetições foi mais eficiente para o controle genético em nível de médias de progênies do que um número maior de plantas por parcela. O coeficiente de herdabilidade é calculado considerando as variâncias genotípicas e ambientais, e desta forma o EXP2 apresentou uma combinação de fatores que propiciou uma melhora na estimativa desse parâmetro, seja pela redução das variâncias residuais, ou pelo aumento das variâncias genotípicas. Os resultados encontrados para $h^{2}$ ne neste trabalho, especialmente no EXP2, foram mais altos do que os encontrados por Costa et al. (2012) ao fazerem avaliação genética de Eucalyptus camaldulensis no estado de Mato Grosso.

A acurácia da seleção de progênies $\left(A c_{\text {prog }}\right.$ ) ou relação entre os valores genéticos preditos e os verdadeiros, foi adequada para todas as variáveis nos dois experimentos. Acurácia superior a 50\%, conforme Resende (2007), pode ser considerada como ideal indicando uma boa precisão na seleção dos genótipos. Ao comparar os dois experimentos, para todas as variáveis, o EXP2 apresentou valores mais elevados de $A c_{\text {prog }}$ em relação ao EXP1 (Tabela 1), indicando que o experimento com uma planta por parcela apresentou melhor precisão no acesso à variação genética verdadeira a partir da variação fenotípica observada.

A média geral não variou muito entre os dois experimentos. No entanto, a amplitude dos valores genotípicos foi expressivamente maior no EXP2 do que no EXP1 para os caracteres de crescimento (Tabela 1), mais uma vez corroborando que o tamanho da parcela não influencia a forma do fuste, mas tem efeito sobre os caracteres de crescimento, gerando maiores amplitudes e variâncias genéticas aliadas a menores variâncias experimentais, refletindo em ganhos genéticos mais promissores e com acurácias superiores em parcelas de uma planta.

O efeito promissor da adoção de experimentos com parcelas menores e maior número de repetições, fica evidente ao avaliar o ganho de seleção (GS e GS\%). Nota-se que o EXP2 destacou-se para os caracteres de crescimento, apresentando superioridade de 5,61 (DAP), 3,01 (altura) e 15,91 (volume) pontos percentuais para GS\%, chegando a ser aproximadamente o dobro para os caracteres DAP e volume, em relação ao EXP1 (Tabela 1). Estes resultados indicam que o experimento com uma planta por parcela favoreceu os indivíduos superiores de uma forma geral, refletido nos parâmetros de ganho de seleção e amplitude.

O tamanho efetivo populacional estimado no EXP1 variou de 226,97 (volume) a 246,46 indivíduos (forma de fuste), e no EXP2 de 235,73 (altura) a 250,60 indivíduos (volume) (Tabela 1). Um número efetivo $\left(N_{e}\right)$ igual a 60 é considerado adequado para obtenção de ganhos nos próximos ciclos seletivos (RESENDE, 1990), maior que 30 é considerado satisfatório (PEREIRA; VENCOVSKY, 1988) e entre 50 e 60 é o recomendado pela EMBRAPA (1991). O resultado aqui obtido é altamente satisfatório para manutenção dos ganhos ao longo dos ciclos de seleção.

$\mathrm{Na}$ análise conjunta dos dois experimentos, o coeficiente de determinação dos efeitos da interação genótipos $\mathrm{x}$ ambientes $\left(c^{2}{ }_{\text {int }}\right)$, para todas as variáveis analisadas, ficou abaixo de 1,5\%, ocasionando estimativas elevadas de correlação genotípica entre o desempenho das progênies nos dois 
Silva et al. - Influência do tamanho de parcelas experimentais

na seleção de progênies de E. camaldulensis Dehnh

experimentos $\left(r_{g l o c}\right)$ (Tabela 1). Isto indica que a interação ocorrida foi, na maioria dos casos, do tipo simples, ou seja, não houve mudança considerável no valor genotípico das progênies nos diferentes delineamentos, indicando que a alteração no tamanho da parcela interferiu apenas no desenvolvimento e nas estimativas de parâmetros genéticos dos indivíduos, não influenciando na seleção de famílias.

A forte correlação genotípica não refletiu na correlação de ordenamento das famílias, representado pelo coeficiente de correlação de Spearman $(\rho)$ entre a classificação das famílias nos dois experimentos. Este coeficiente sempre propiciou correlações positivas, mas em magnitudes inferiores as correlações genotípicas entre as famílias, sendo que para forma de fuste a correlação de Spearman foi inferior a metade da correlação genotípica, esta proporção manteve-se praticamente a mesma para as demais características avaliadas. O número de famílias coincidentes entre as trinta melhores (FC) também indica algumas trocas de posições, sendo que13 famílias foram coincidentes entre as melhores para forma de fuste, 17 para DAP, 15 para altura e 16 para volume total das árvores (Tabela 1), refletindo que o tamanho de parcelas interfere no ordenamento das famílias selecionadas. No entanto o número total de famílias selecionadas em todos os caracteres foram semelhantes e a coincidência entre essas famílias selecionadas também foi alta.

\section{CONCLUSÕES}

- O tamanho e forma de parcelas não interfere na forma de fuste, mas influencia substancialmente os caracteres de crescimento;

- Experimentos com menor número de plantas por parcelas e maior números de repetições permitem obter maiores estimativas de variância genotípica e/ou menores variâncias ambientais, proporcionando melhores coeficientes de herdabilidade e acurácia seletiva;

- O experimento com uma planta por parcela é mais eficiente na seleção de progênies de E. camaldulensis, proporcionando ganhos superiores para caracteres de crescimento e similares para forma de fuste, sendo recomendado para testes de progênies do gênero Eucalyptus.

\section{REFERÊNCIAS BIBLIOGRÁFICAS}

ARANTES, F. C.; GONÇALVES, P. S.; SCALOPPI JR., E. J.; MORAES, M. L. T.; RESENDE, M. D. V. Ganho genético com base no tamanho efetivo populacional de progênies de seringueira. Pesquisa Agropecuária Brasileira. Brasília, v. 45, n. 12, p. 1419-1424, 2010.

ARAÚJO, M. J.; DIAS, D. C.; SCARPINATI, E. A.; PAULA, R. C. Número de repetições, de plantas por parcela e de avaliações para testes clonais de eucalipto. Pesquisa agropecuária brasileira, Brasília, v. 50, n. 10, p. 923931, 2015.

BAUER, A. M.; REETZ, T. C.; LÉON, J. Estimation of breeding values of inbred lines using best linear unbiased prediction (BLUP) and genetic similarities. Crop Science, Madison, v. 46, p. 2685-2691, 2006.

BOOTH, T. H. Eucalypt plantations and climate change. Forest Ecology and Management, Amsterdam, v. 301, p. 28-34, 2013.

COSTA, R. B.; AZEVEDO, L. P. A.; MARTINEZ, D. T.; TSUKAMOTO FILHO, A. A.; FERNANDES, D. A.; OLIVEIRA, O. E.; RESENDE, M. D. V. Avaliação genética de Eucalyptus camaldulensis no Estado de Mato Grosso. Pesquisa Florestal Brasileira, Colombo, v. 32, n. 70, p. 165-173, 2012.

DIAS, T. C. Forma e tamanho ótimos de parcelas experimentais de eucalipto clonal em sistema de integração lavoura-pecuária-floresta. 2014. 114 p. Dissertação (Mestrado em Engenharia Florestal). Universidade Federal de Lavras, Lavras, 2014.

ELDRIDGE, K.; DAVIDSON, J.; HARWOOD, C.; WYK, G. Eucalypt domestication and breeding. Oxford: Clarendon Press, 1993. 288 p. 
EMBRAPA Relatório de cooperação técnica (EMBRAPA/CENIBRA) em melhoramento genético de Eucalyptus grandis. Colombo: CNPF, 1991. 20 p. (Não publicado).

FARIAS NETO, J. T.; LINS, P. M. P.; RESENDE, M. D. V.; MULLER, A. A. Seleção genética em progênies híbridas de coqueiro. Revista Brasileira de Fruticultura, Jaboticabal, v. 31, n. 1, p. 190-196, 2009.

GOLFARI, L.; CASER, R. L., MOURA, V. P. G. Zoneamento ecológico esquemático para reflorestamento no Brasil - $2^{\mathrm{a}}$ aproximação. Belo Horizonte: PRODEPEF, 1978. 66 p. (Série Técnica, 11).

IBA - INDÚSTRIA BRASILEIRA DE ÁRVORES. Anuário estatístico da indústria brasileira de árvores: ano base 2014. Brasília, 2015. 97 p. Disponível em: <http://www.iba.org/shared/iba 2015.pdf>. Acesso em: 20 jan. 2015.

MASSARO, R. A. M.; BONINE, C. A. V.; SCARPINATI, E. A.; PAULA, R. C. Viabilidade de aplicação da seleção precoce em testes clonais de Eucalyptus spp. Ciência Florestal, Santa Maria, v. 20, n. 4, p. 597-609, out./dez. 2010

OLIVEIRA, G. M. V.; MELLO, J. M.; LIMA, R. R.; SCOLFORO, J. R. S.; OLIVEIRA, A. D. Tamanho e forma de parcelas experimentais para Eremanthus erythropappus. Cerne, Lavras, v. 17, n. 3, p. 327-338, jul./set. 2011.

PAVAN, B. E.; PAULA, R. C.; PERECIN, D.; CANDIDO, L. S.; SCARPINATI, E. A. Efeito competicional em testes de progênies de eucalipto. Ciência Florestal, Santa Maria, v. 22, n. 3, p. 433-443. 2012.

PAVAN, B. E.; PAULA, R. C.; PERECIN, D.; CANDIDO, L. S.; SCARPINATI, E. A.; CANDIDO, L. S. Early selection in open-pollinated Eucalyptus families based on competition covariates. Pesquisa Agropecuária Brasileira, Brasília, v. 49, n. 6, p. 483-492, jun. 2014.

PEREIRA, M. B.; VENCOVSKY, R. Limites da seleção recorrente: 1- fatores que afetam o acréscimo das freqüências alélicas. Pesquisa Agropecuária Brasileira, Brasília, v. 23, n. 7, p. 769-780, 1988.

PINTO, D. S.; RESENDE, R. T.; MESQUITA, A. G. G.; ROSADO, A. M. CRUZ, C. D. Seleção precoce para características de crescimento em testes clonais de Eucalyptus urophylla. Scientia Forestalis, Piracicaba, v. 42, n. 102, p. 251-257, jun. 2014.

PUPIN, S.; ARIELEN SANTOS, V. A.; ZARUMA, D. U. G.; MIRANDA, A. C.; SILVA, P. H. M.; MARINO, C. L.; SEBBENN, A. M.; MORAES, M. L. T. Produtividade, estabilidade e adaptabilidade em progênies de polinização aberta de Eucalyptus urophylla S.T. Blake. Scientia Forestalis. Piracicaba, v. 43, n. 105, p. 127-134, mar. 2015.

QUISEN, R. C. Transformação genética de Eucalyptus camaldulensis via co-cultivo com Agrobacterium tumefaciens. 2007. 125 p Tese (Doutorado em Agronomia) - Universidade Federal do Paraná, Curitiba, 2007.

RESENDE, M. D. V. Delineamento de experimentos de seleção para maximização da acurácia seletiva e do progresso genético. Revista árvore, Viçosa, v. 19, n. 4, p. 470-500, 1995.

RESENDE，M. D. V. SELEGEN-REML/BLUP: Sistema estatístico e seleção genética computadorizada via modelos lineares mistos. Colombo: Embrapa Florestas, 2007. 359 p.

RESENDE, M. D. V.; DUARTE, J. B. Precisão e controle de qualidade em experimentos de avaliação de cultivares. Pesquisa Agropecuária Tropical, Goiânia, v. 37, n. 3, p. 182-194, 2007.

RESENDE, M. D. V.; HIGA, A. R. Estratégias de melhoramento para eucaliptos visando a seleção de híbridos. Boletim Pesquisa Florestal, Colombo, n. 21, p. 49-60, 1990.

SCARPINATI, E. A.; PERECIN, D.; PAULA, R. C.; VALENCISE, C. A.; PAVAN, B. E.; CANDIDO, L. S.; Influência do modelo de análise estatística e da forma das parcelas experimentais na seleção de clones de Eucalyptus spp. Revista Árvore, Viçosa, v. 33, n. 4, p. 769-776, 2009. 
Silva et al. - Influência do tamanho de parcelas experimentais

na seleção de progênies de E. camaldulensis Dehnh

SEBBENN, A. M.; SIQUEIRA, A. C. M. F.; KAGEYAMA, P. Y.; MACHADO, J. A. R. Parâmetros genéticos na conservação da cabreúva - Myroxylon peruiferum L.F. Allemão. Scientia Forestalis, Piracicaba, n. 53, p. 3138, 1998.

SILVA, J. M. Avaliação da variabilidade genética em uma população base de Eucalyptus camaldulensis Dehnh. para fins de conservação e melhoramento genético. 2010. 153 p Tese (Doutorado em Agronomia). Universidade Estadual Paulista, Ilha Solteira, 2010.

SILVA, R. L.; XAVIER, A.; LEITE, H. G.; PIRES, I. E. Determinação do tamanho ótimo da parcela experimental pelos métodos da máxima curvatura modificado, do Coeficiente de correlação intraclasse e da análise visual em testes clonais de eucalipto. Revista Árvore, Viçosa, v. 27, n. 5, p. 669-676, 2003.

VENCOVSKY, R.; BARRIGA, P. Genética biométrica no fito-melhoramento. Ribeirão Preto: SBG, 1992.496 p.

VERARDI, C. K.; RESENDE, M. D. V.; COSTA, R. B.; GONÇALVES, P. S. Adaptabilidade e estabilidade da produção de borracha e seleção em progênies de seringueira. Pesquisa Agropecuária Brasileira, Brasília, v. 44, n. 10, p. 1277-1282, 2009.

Recebido em 04/01/2016

Aceito para publicação em 15/06/2016 